\title{
Nonlinear Analysis of Structures Cable - Truss
}

\author{
Ever Coarita and Leonardo Flores
}

\begin{abstract}
This paper aims to propose a mixed algorithm to simulate the interaction between cable-truss; the study is focused on the geometric nonlinear analysis since the cables always have nonlinear behavior under static loads.

By using lagrangian formulations, it is determined the nonlinear stiffness matrices from the elements cable and truss, which are assembled to a structure on a global reference system. The balance of the structures under the action of external loads is solved by an iterative incremental method, which highlights the secant method; which gets better approaches to the solution with a smaller increase in loads. The solution algorithms are implemented with matlab.
\end{abstract}

Index Terms - Cable, nonlinear, truss.

\section{INTRODUCTION}

Many structures in the initial state have a linear behavior; however, there are exceptions where the relationship between force and displacement cannot be described properly with a model of linear behavior of the structure. The sources of non-linearity are due to the nonlinear behavior of the material, the geometric nonlinearity or a combined effect of these [1].

Among the exceptions, the cables always have a nonlinear behavior with a nonlinearity due to only having a tensional behavior and geometric nonlinearity [2]. In general, cable structures exhibit a flexibility that cannot be attributed to low axial stiffness of the elements; but the geometry of the structure [3], in many cases are structures that has large displacements, for that reason it becomes necessary their study in the nonlinear range.

The usual methods of analysis of cable structures are based on the discretization of the cable into smaller elements to formulate the equilibrium equations. Then, numerical methods used to solve the resulting equations or elements with appropriate values of Young modulus are applied. In the present paper the cable structures, dividing them into cable stiffness matrix elements deduced from the equation of the catenary are studied.

\section{A. Antecedent}

The study of the cable element has been of great interest for many years. Thus Galileo 1638 says that the form of a trailing cable is parabolic, this analogy to the flight of a projectile. In 1691 the Bernoulli, Leibnitz and Huygens brothers concluded that it has the shape of the catenary [4] and they found the equilibrium equations for inextensible cable. Leibnitz use calculus theory to derive the equation of this curve [5].

Manuscript received April 5, 2014; July 11, 2014.

The authors are with University National of Engineering of Peru, Puno (e-mail: dencoarita@hotmail.com, leo_flo_gon_2005@yahoo.com)
The Bernoulli brothers also made the general differential equation of equilibrium of a chain element, under various loads that considers the effects of incorporating stretching Hooke's Law. In 1891, Routh solved the equation for an elastic symmetrically suspended catenary comprised of a linear elastic material [6], while the previous job Feld extended to not symmetrical case, but unfortunately the coordinates used in both solutions made their application difficult [4].

In 1981 Irvine, to avoid this difficulty, adopts a Lagrangian approach to the solution of the elastic catenary obtaining an expression for the tangent stiffness matrix of a non-symmetrical elastic cable.

Among the various methodologies that have been proposed to model cable structures, these are represented using two different methods.

The first method is based on interpolation polynomial functions to describe the shape and displacement; this is a common method in the development of finite element analysis. In this context, the two nodes straight element is element used in modeling cables [5]. This element has only axial stiffness and it is generally applicable to pre-stressed cables. When there are a large curvature loose cables, presenting its geometry is performed by a large number of bar elements; analysis becomes inefficient while there are more degrees of freedom. When you want to model the loose cable, the modulus of elasticity is replaced by an equivalent module, which takes into account the flexible cable, this rigid as was proposed by Ernst [5]. Usually in a cable that has relatively high stresses and short length, the approximate equivalent module is recommended for good results [7]. Another model is the multi-node isoperimetric element, which is achieved by adding more nodes to the finite element; the elements are usually three or four nodes, which use parabolic interpolation functions or cubes respectively. Such cables work well with small deflections, but for large deflections must use more elements derived continuity between elements is not met [8]. The continuity of the derivative can be accomplished by adding degrees of rotational freedom to the nodes. This model is known as curved elements with degrees of free rotation, and this element was developed by Gambhir and Batchelor [5], those using cubic polynomials which describe the displacement and shape of the cable. Therefore, the elements based on polynomial interpolation are generally appropriate to model cables with small vertical deflections, as mentioned above, cable elements with large vertical deflections many elements are employed for the curved geometry of the cable, which requires a large number of arithmetic operations. This influences the solution time of the problem [8].

The second method uses analytical formulations taking into account effects of applied load along the cable, and 
describes the real behavior of the cable. In this context, the parabolic elastic element is generally used in the cable structure analysis, due to its simple shape compared to the catenary [5]. Another model is the elastic associated catenary, which is an extension of Irvine model developed by Ahmadi-Kashani and Bell [2]. In this model, a constant load per unit length applied is considered although the cable elongation. The positive elongation is produced by an increase of the total load carried and this usable model for cables subjected to external pressures such as wind, snow or other external forces [6]. In this paper it is considered an elastic catenary element, which is accurate because the equilibrium configuration of a hanging catenary cable is nature [7] and this model is appropriate for a perfectly flexible cable that is subject to self-weight loads [5]. The elastic element catenary formulation is based on the exact analytic solution to the elastic catenary developed by O'Brien [7]. In this method, only two nodes catenary element without internal joints are necessary to model a single cable, this element can be used for modeling small and large vertical and horizontal deflections. In this way the cable can be represented by a single element, which has certain advantages, such as reduced number of degrees of freedom and considering the elastic cable nonlinear effect in all cases [8]. Because of the analytical expressions is continuity preserved through the element boundaries.

Another model used is the inextensible catenary element, which is infinitely axially rigid element $(A E \rightarrow \infty)$ which prevents increase in length, perfectly flexible $\left(E I_{n}=0\right)$, free of torsional rigidity and capable of supporting only tensional forces [5]. The applications based in this type of elements find some difficulties because the curve that relates force and displacement is asymptotic, so they tend to experience high numerical instability, accusing very difficult or even impossible convergence [6].

\section{THEORETICAL BASIS}

\section{A. Element Type Truss}

\section{1) Variational formulation of the element type truss}

The equilibrium equations according to the principle of virtual work can be written to the body in nonlinear incremental way [9]:

$$
\int_{V} \delta \varepsilon^{T} E \varepsilon d V+\int_{V} \delta \eta^{T} \tau d V+{ }^{1} R={ }^{2} R
$$

Decomposing increasing deformation $\varepsilon$ in its linear portion (e) and nonlinear part $(\eta)$, the equation can be expressed as:

$$
\begin{gathered}
\int_{V} \delta e^{T} E e d V+\int_{V} \delta e^{T} E \eta d V+\int_{V} \delta \eta^{T} E e d V \\
+\int_{V} \delta \eta^{T} E \eta d V .+\int_{V} \delta \eta^{T} \tau d V .+{ }^{1} R={ }^{2} R
\end{gathered}
$$

For the space truss element as in Fig. 1, displacement $(u, v, w)$ may be related to the displacement $\left(u_{i}, v_{i}, w_{i}\right)$ and $\left(u_{j}, v_{j}, w_{j}\right)$ of the two ends of the element used linear interpolation functions such as [9]:

$$
\begin{aligned}
& u=u_{i}\left(1-\frac{x}{L}\right)+u_{j}\left(\frac{x}{L}\right) \\
& v=v_{i}\left(1-\frac{x}{L}\right)+v_{j}\left(\frac{x}{L}\right) \\
& w=w_{i}\left(1-\frac{x}{L}\right)+w_{j}\left(\frac{x}{L}\right)
\end{aligned}
$$

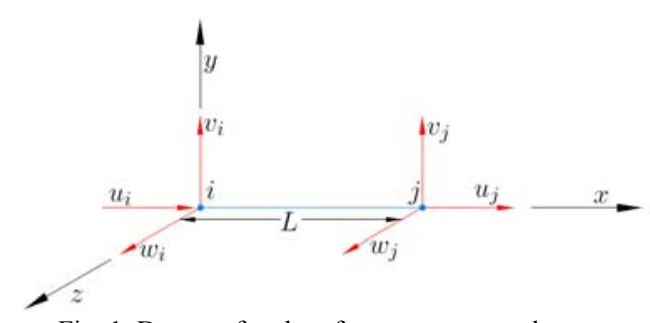

Fig. 1. Degrees freedom for a space truss element.

The balance equation is expressed as:

$$
\left(k_{e}+k_{g}+s_{1}+s_{2}+s_{3}\right) u+{ }^{1} f={ }^{2} f
$$

where:

$k_{e}$ is the elastic stiffness matrix:

$$
k_{e}=\frac{E A}{L}\left[\begin{array}{cccccc}
1 & 0 & 0 & -1 & 0 & 0 \\
0 & 0 & 0 & 0 & 0 & 0 \\
0 & 0 & 0 & 0 & 0 & 0 \\
-1 & 0 & 0 & 0 & 0 & 0 \\
0 & 0 & 0 & 0 & 0 & 0 \\
0 & 0 & 0 & 0 & 0 & 0
\end{array}\right]
$$

$k_{g}$ is the geometric stiffness matrix: and the matrices $s_{1}$, $s_{2}$ and $s_{3}$ of higher order are:

$$
\begin{aligned}
& k_{g}=\frac{F}{L}\left[\begin{array}{cccccc}
1 & 0 & 0 & -1 & 0 & 0 \\
0 & 1 & 0 & 0 & -1 & 0 \\
0 & 0 & 1 & 0 & 0 & -1 \\
-1 & 0 & 0 & 1 & 0 & 0 \\
0 & -1 & 0 & 0 & 1 & 0 \\
0 & 0 & -1 & 0 & 0 & 1
\end{array}\right] \\
& s_{1}=\frac{E A}{2 L^{2}}\left[\begin{array}{cccccc}
\Delta u & \Delta v & \Delta w & -\Delta u & -\Delta v & -\Delta w \\
0 & 0 & 0 & 0 & 0 & 0 \\
0 & 0 & 0 & 0 & 0 & 0 \\
-\Delta u & -\Delta v & -\Delta w & \Delta u & \Delta v & \Delta w \\
0 & 0 & 0 & 0 & 0 & 0 \\
0 & 0 & 0 & 0 & 0 & 0
\end{array}\right] \\
& s_{2}=\frac{E A}{2 L^{2}}\left[\begin{array}{cccccc}
2 \Delta u & 0 & 0 & -2 \Delta u & 0 & 0 \\
\Delta v & \Delta u & 0 & -\Delta v & -\Delta u & 0 \\
\Delta w & 0 & \Delta u & -\Delta w & 0 & -\Delta u \\
-2 \Delta u & 0 & 0 & 2 \Delta u & 0 & 0 \\
-\Delta v & -\Delta u & 0 & \Delta v & \Delta u & 0 \\
-\Delta w & 0 & -\Delta u & \Delta w & 0 & \Delta u
\end{array}\right] \\
& s_{3}=\frac{E A}{6 L^{3}}\left[\begin{array}{cc}
h & -h \\
-h & h
\end{array}\right]
\end{aligned}
$$


where:

$h=\left[\begin{array}{ccc}3 \Delta u^{2}+\Delta v^{2}+\Delta w^{2} & 2 \Delta u \Delta v & 2 \Delta u \Delta w \\ 2 \Delta u \Delta v & \Delta u^{2}+3 \Delta v^{2}+\Delta w^{2} & 2 \Delta v \Delta w \\ 2 \Delta u \Delta w & 2 \Delta v \Delta w & \Delta u^{2}+\Delta v^{2}+3 \Delta w^{2}\end{array}\right]$

\section{B. Element Type Cable}

\section{1) Hypothesis element type cable}

The cross section is constant before and after the deformation of the element.

The element is supposed to be perfectly flexible and acts only stressed forces.

Conservation of the mass of cable element after deformation by varying the weight per unit length for each cable elongation.

\section{2) Lagrangian formulation of the element type cable}

The cable is considered an initial length $L_{o}$, suspended between two fixed supports $I$ and $J$, which are Cartesian coordinates $(0,0,0)$ and $\left(l_{x}, l_{y}, l_{z}\right)$ respectively. Thus, the horizontal projections of its length are $l_{x}$ and $l_{y}$, while on the vertical projection is $l_{z}$. Be the point $P$ an internal point of the cable element. When the cable is deformed by action of its self-weight $\left(w L_{o}\right)$, the point $P$ moves into a new position described by the Cartesian coordinates $(x, y, z)$ and the Lagrangian coordinate $p$, by what is defined as the segment length deformed between the origin and the point on the deformed geometry corresponding to the point $P$ on the original geometry [10], as shown in Fig. 2.

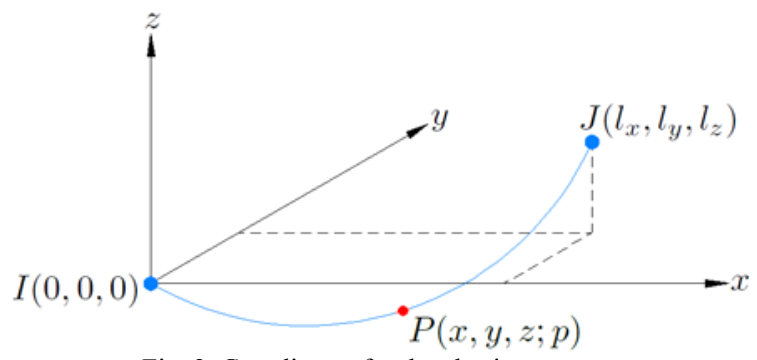

Fig. 2. Coordinates for the elastic catenary.

The geometric constraint that must be met by force balanced is:

$$
\left(\frac{d x}{d p}\right)^{2}+\left(\frac{d y}{d p}\right)^{2}+\left(\frac{d z}{d p}\right)^{2}=1
$$

Prestressed cable $T$, is related to the strain $\varepsilon$ and Hooke's law:

$$
T=E A \mathcal{E}=E A\left(\frac{d p-d s}{d s}\right)=E A\left(\frac{d p}{d s}-1\right)
$$

where $E$ is the elastic modulus, $A$ is the constant cross-sectional area in the undeformed profile.

The conditions at the ends of the nodes are:

$$
\begin{gathered}
x=0, y=0, z=0, p=0 \text { in } s=0 \\
x=l_{x}, y=l_{y}, z=l_{z}, p=L \text { in } s=L_{o}
\end{gathered}
$$

where $L$ is the length in the deformed profile.

The Lagrangian $s$ and the Cartesian coordinates are related as shown in the equations (12a), (12b) and (12c):

$$
\begin{aligned}
& x(s)=\int_{s} \frac{d x}{d s} d s=\int_{s} \frac{d x}{d p} \frac{d p}{d s} d s \\
& y(s)=\int_{s} \frac{d y}{d s} d s=\int_{s} \frac{d y}{d p} \frac{d p}{d s} d s \\
& z(s)=\int_{s} \frac{d z}{d s} d s=\int_{s} \frac{d z}{d p} \frac{d p}{d s} d s
\end{aligned}
$$

By integrating in Cartesian coordinates $x, y$ and $z$ takes depending on the length of the deformed segment:

$$
\begin{array}{r}
x(s)=-\frac{F_{1} s}{E A}-\frac{F_{1}}{w}\left\{\ln \left(\sqrt{F_{1}^{2}+F_{2}^{2}+\left(w s-F_{3}\right)^{2}}+w s-F_{3}\right)\right. \\
-\ln \left(\sqrt{\left.\left.F_{1}^{2}+F_{2}^{2}+F_{3}^{2}-F_{3}\right)\right\}}\right. \\
y(s)=-\frac{F_{2} s}{E A}-\frac{F_{2}}{w}\left\{\operatorname { l n } \left(\sqrt{\left.F_{1}^{2}+F_{2}^{2}+\left(w s-F_{3}\right)^{2}+w s-F_{3}\right)}\right.\right. \\
-\ln \left(\sqrt{\left.\left.F_{1}^{2}+F_{2}^{2}+F_{3}^{2}-F_{3}\right)\right\}}\right. \\
z(s)=-\frac{F_{3} s}{E A}+\frac{w s^{2}}{2 E A}+\frac{1}{w}\left\{\sqrt{F_{1}^{2}+F_{2}^{2}+\left(w s-F_{3}\right)^{2}}\right. \\
\left.-{\sqrt{F_{1}^{2}+F_{2}^{2}+F_{3}}}^{2}\right\}
\end{array}
$$

By including boundary conditions, we have:

$$
\begin{aligned}
& l_{x}=-\frac{F_{1} L_{o}}{E A}-\frac{F_{1}}{w}\left\{\ln \left(\sqrt{F_{1}^{2}+F_{2}^{2}+\left(w L_{o}-F_{3}\right)^{2}}+w L_{o}-F_{3}\right)\right. \\
& \left.-\ln \left({\sqrt{F_{1}}}^{2}+F_{2}^{2}+F_{3}^{2}-F_{3}\right)\right\}
\end{aligned}
$$

$$
\begin{gathered}
l_{y}=-\frac{F_{2} L_{o}}{E A}-\frac{F_{2}}{w}\left\{\ln \left(\sqrt{F_{1}^{2}}+F_{2}^{2}+\left(w L_{o}-F_{3}\right)^{2}+w L_{o}-F_{3}\right)\right. \\
\left.-\ln \left(\sqrt{F_{1}^{2}+F_{2}^{2}+F_{3}^{2}}-F_{3}\right)\right\} \\
l_{z}=-\frac{F_{3} L_{o}}{E A}+\frac{w L_{o}^{2}}{2 E A}+\frac{1}{w}\left\{\sqrt{F_{1}^{2}+F_{2}^{2}+\left(w L_{o}-F_{3}\right)^{2}}\right. \\
\left.-\sqrt{F_{1}^{2}+F_{2}^{2}+F_{3}^{2}}\right\}
\end{gathered}
$$

Since $l_{x}, l_{y}$ and $l_{z}$ are written in terms of end forces $I\left(F_{1}, F_{2}, F_{3}\right)$, these can be expressed in small variations thereof by a first order linearization to approximate [11]:

$$
\begin{array}{r}
d l_{x}=\frac{\partial l_{x}}{\partial F_{1}} d F_{1}+\frac{\partial l_{x}}{\partial F_{2}} d F_{2}+\frac{\partial l_{x}}{\partial F_{3}} d F_{3} \\
d l_{y}=\frac{\partial l_{y}}{\partial F_{1}} d F_{1}+\frac{\partial l_{y}}{\partial F_{2}} d F_{2}+\frac{\partial l_{y}}{\partial F_{3}} d F_{3}
\end{array}
$$




$$
d l_{z}=\frac{\partial l_{z}}{\partial F_{1}} d F_{1}+\frac{\partial l_{z}}{\partial F_{2}} d F_{2}+\frac{\partial l_{z}}{\partial F_{3}} d F_{3}
$$

Or expressed in matrix form:

$$
\left\{\begin{array}{l}
d l_{x} \\
d l_{y} \\
d l_{z}
\end{array}\right\}=\left[\begin{array}{lll}
f_{11} & f_{12} & f_{13} \\
f_{21} & f_{22} & f_{23} \\
f_{31} & f_{32} & f_{33}
\end{array}\right]\left\{\begin{array}{l}
d F_{1} \\
d F_{2} \\
d F_{3}
\end{array}\right\}=F\left\{\begin{array}{l}
d F_{1} \\
d F_{2} \\
d F_{3}
\end{array}\right\}
$$

where $F$ is the incremental flexibility matrix, whose elements $f_{i j}$ are given as follows:

$$
\begin{gathered}
f_{11}=-\left(\frac{L_{o}}{E A}+\frac{1}{w} \ln \frac{T_{j}+F_{6}}{T_{i}-F_{3}}\right)+\frac{F_{1}^{2}}{w}\left[\frac{1}{T_{i}\left(T_{i}-F_{3}\right)}+\frac{1}{T_{j}\left(T_{j}-F_{6}\right)}\right] \\
f_{12}=f_{21}=\frac{F_{1} F_{2}}{w}\left[\frac{1}{T_{i}\left(T_{i}-F_{3}\right)}+\frac{1}{T_{j}\left(T_{j}-F_{6}\right)}\right] \\
f_{13}=f_{31}=\frac{F_{1}}{w}\left[\frac{1}{T_{j}}-\frac{1}{T_{i}}\right] \\
f_{22}=-\left(\frac{L_{o}}{E A}+\frac{1}{w} \ln \frac{T_{j}+F_{6}}{T_{i}-F_{3}}\right)+\frac{F_{2}^{2}}{w}\left[\frac{1}{T_{i}\left(T_{i}-F_{3}\right)}+\frac{1}{T_{j}\left(T_{j}-F_{6}\right)}\right] \\
f_{23}=f_{32}=\frac{F_{2}}{w}\left[\frac{1}{T_{j}}-\frac{1}{T_{i}}\right] \\
f_{33}=-\frac{L_{o}}{E A}-\frac{1}{w}\left[\frac{F_{6}}{T_{j}}+\frac{F_{3}}{T_{i}}\right]
\end{gathered}
$$

where $T_{i}$ and $T_{j}$ are the cable tension at nodes $I$ and $J$ respectively. The nodal forces $F_{1}, F_{2}, F_{3}, F_{4}, F_{5}, F_{6}, T_{i}$ and $T_{j}$ are related as:

$$
\begin{gathered}
F_{4}=-F_{1} \\
F_{5}=-F_{2} \\
F_{6}=-F_{3}+w L_{o} \\
T_{i}=\sqrt{F_{1}^{2}+F_{2}^{2}+F_{3}^{2}} \\
T_{j}=\sqrt{F_{4}^{2}+F_{5}^{2}+F_{6}^{2}}
\end{gathered}
$$

The cable stiffness matrix is obtained by inverting the flexibility matrix $F$ as:

$$
K_{c}=F^{-1}=\left[\begin{array}{lll}
f_{11} & f_{12} & f_{13} \\
f_{21} & f_{22} & f_{23} \\
f_{31} & f_{32} & f_{33}
\end{array}\right]^{-1}
$$

The tangent stiffness matrix and the corresponding vector element internal forces of the cable, expressed in terms of six degrees of freedom [8]:

$$
\begin{gathered}
K_{c t}=\left[\begin{array}{rr}
-K_{c} & K_{c} \\
K_{c} & -K_{c}
\end{array}\right] \\
f_{\text {int }}=\left\{\begin{array}{llllll}
F_{1} & F_{2} & F_{3} & F_{4} & F_{5} & F_{6}
\end{array}\right\}^{T}
\end{gathered}
$$

\section{Cable Element Algorithm}

To determine the tangent stiffness matri $x K_{c t}$, must first determine the $F_{1}, F_{1}$ and $F_{3}$ forces $I$ node [12]. To do this, we start with an initial estimate of these nodal forces, through [8]:

$$
\begin{gathered}
F_{1}=-\frac{w l_{x}}{2 \lambda} \\
F_{2}=-\frac{w l_{y}}{2 \lambda} \\
F_{3}=\frac{w}{2}\left(-l_{z} \frac{\cosh \lambda}{\operatorname{senh} \lambda}+L_{o}\right)
\end{gathered}
$$

where:

$$
\lambda=\left\{\begin{array}{cc} 
& \left(l_{x}^{2}+l_{y}^{2}\right)=0 \\
10^{6} & L_{o}^{2} \leq\left(l_{x}^{2}+l_{y}^{2}+l_{z}^{2}\right) \\
0.2 & L_{o}^{2}>\left(l_{x}^{2}+l_{y}^{2}+l_{z}^{2}\right)
\end{array}\right.
$$

By reemplacing the estimated forces (16), the coordinates $\left(l_{x o}, l_{y o}, l_{z o}\right)$ of the cable is determined and compared to the indicated coordinate of the node $J\left(l_{x}, l_{y}, l_{z}\right)$, the difference vector is determined $\Delta L=\left\{\begin{array}{lll}\left(l_{x o}-l_{x}\right) & \left(l_{y o}-l_{y}\right) & \left(l_{z o}-l_{z}\right)\end{array}\right\}^{T}$, which should be less than the requested tolerance.

If the tolerance is not met, the approach forces from $I$ node is corrected using the difference vector as:

$$
\left\{\begin{array}{l}
\Delta F_{1} \\
\Delta F_{2} \\
\Delta F_{3}
\end{array}\right\}=K_{c}\left\{\begin{array}{l}
\Delta l_{x} \\
\Delta l_{y} \\
\Delta l_{z}
\end{array}\right\}
$$

Forces are then updated:

$$
\left\{\begin{array}{l}
F_{1} \\
F_{2} \\
F_{3}
\end{array}\right\}=\left\{\begin{array}{l}
F_{1} \\
F_{2} \\
F_{3}
\end{array}\right\}+\left\{\begin{array}{l}
\Delta F_{1} \\
\Delta F_{2} \\
\Delta F_{3}
\end{array}\right\}
$$

where $K_{c}$ is the stiffness matrix of equation (22).

Below the algorithm used is supposed to meet the stiffness matrix of a cable element.

Step 1: Initial data are the coordinates of the nodes $I\left(x_{i}, y_{i}, z_{i}\right)$ and $J\left(x_{j}, y_{j}, z_{j}\right)$, physical properties $(E, A$, $\left.w, L_{o}\right)$ and tolerance tol .

Step 2: Calculate the relative coordinates between nodes $I \quad$ and $J: l_{x 0}=x_{j}-x_{i}, l_{y 0}=y_{j}-y_{i}$ and $l_{z 0}=z_{j}-z_{i}$. 
Step 3: Give an initial approximation of the forces $F_{1}$, $F_{2}, F_{3}$ node $I$, using equation (25).

Step 4: Update the relative coordinate of the node $J$ $\left(l_{x}, l_{y}, l_{z}\right)$, replacing $F_{1}, F_{2}$ and $F_{3}$ in equation (16).

Step 5: Calculate the difference vector:

$$
\Delta L=\left\{\begin{array}{l}
l_{x o}-l_{x} \\
l_{y o}-l_{y} \\
l_{z o}-l_{z}
\end{array}\right\}=\left\{\begin{array}{l}
\Delta l_{x} \\
\Delta l_{y} \\
\Delta l_{z}
\end{array}\right\}
$$

Step 6: If $\|\Delta L\|_{\infty}<t o l$, calculate $K_{c t}$ and $F_{\text {int }}$ with (23) and (24) respectively. Otherwise continue the following steps.

Step 7: Calculate the correction vector node forces $I$ : $\Delta F=K_{c} \Delta L$ (27).

Step 8: Refresh forces the next iteration using (28) and return to the Step 4 .

When the initial tension $T_{o}$ cable in the first node is known, the next iteration process is used to determine the length of cable not worked.

Step 1: For initial physical properties $w, E, A$, the stress at node $I\left(T_{o}\right)$ and the coordinates of the nodes $I\left(x_{i}, y_{i}, z_{i}\right)$ and $J\left(x_{j}, y_{j}, z_{j}\right)$ is required.

Step 2: Calculate the relative coordinates between nodes $I \quad$ and $J: \quad l_{x 0}=x_{j}-x_{i}, \quad l_{y 0}=y_{j}-y_{i} \quad$ and $l_{z 0}=z_{j}-z_{i}$.

Step 3: Initialize the unstressed length $L_{o}$ and forces of node $I\left(F_{1}, F_{2}, F_{3}\right)$ as follows:

$$
\begin{gathered}
L_{o}=\sqrt{l_{x o}^{2}+l_{y o}^{2}+l_{z o}^{2}} \\
F_{1}=-\frac{l_{x o}}{L_{o}} T_{o} \\
F_{2}=-\frac{l_{y o}}{L_{o}} T_{o} \\
F_{3}=-\frac{l_{z o}}{L_{o}} T_{o}
\end{gathered}
$$

Step 4: Update the relative coordinate of node $J$ $\left(l_{x}, l_{y}, l_{z}\right)$, replacing $F_{1}, F_{2}, F_{3}$ and $L_{o}$ in equation (16).

Step 5: Calculate the vector difference $\Delta L=\left\{\begin{array}{lll}\left(l_{x o}-l_{x}\right) & \left(l_{y o}-l_{y}\right) & \left(l_{z o}-l_{z}\right)\end{array}\right\}^{T}$ the difference between the know stress $T_{o}$ and the estimated $T_{i}$ (21a): $\Delta T=T_{o}-T_{i}$.

Step 6: If $\|\Delta L\|_{\infty}<t o l$ and $\|\Delta T\|_{\infty}<t o l$, calculate $K_{c t}$ and $F_{\text {int }}$ with (23) and (24) respectively. Otherwise continue with the following Steps.

Step 7: Calculate $C_{c}$ for differentiation of the expressions (16) and (21a) with respect to $F_{1}, F_{2}, F_{3}$ and $L_{o}$ as:

$$
C c=\left[\begin{array}{llll}
\frac{\partial l_{x}}{\partial F_{1}} & \frac{\partial l_{x}}{\partial F_{2}} & \frac{\partial l_{x}}{\partial F_{3}} & \frac{\partial l_{x}}{\partial L_{o}} \\
\frac{\partial l_{y}}{\partial F_{1}} & \frac{\partial l_{y}}{\partial F_{2}} & \frac{\partial l_{y}}{\partial F_{3}} & \frac{\partial l_{y}}{\partial L_{o}} \\
\frac{\partial l_{z}}{\partial F_{1}} & \frac{\partial l_{z}}{\partial F_{2}} & \frac{\partial l_{z}}{\partial F_{3}} & \frac{\partial l_{z}}{\partial L_{o}} \\
\frac{\partial T_{i}}{\partial F_{1}} & \frac{\partial T_{i}}{\partial F_{2}} & \frac{\partial T_{i}}{\partial F_{3}} & \frac{\partial T_{i}}{\partial L_{o}}
\end{array}\right]
$$

Step 8: Calculate the correction vector:

$$
\left\{\begin{array}{l}
\Delta F_{1} \\
\Delta F_{2} \\
\Delta F_{3} \\
\Delta L_{o}
\end{array}\right\}=C_{c}\left\{\begin{array}{l}
\Delta l_{x} \\
\Delta l_{y} \\
\Delta l_{z} \\
\Delta T_{i}
\end{array}\right\}
$$

Step 9: Update forces: $F_{1}=F_{1}+\Delta F_{1}, F_{2}=F_{2}+\Delta F_{2}$, $F_{3}=F_{3}+\Delta F_{3}$, length: $L_{o}=L_{o}+\Delta L_{o}$ and return to Step 4

\section{Description of the Algorithm}

With the algorithms described in the previous section, it is possible to determine the configuration of the cable supported at nodes $I$ and $J$, and whose relative distance is $\left(l_{x o}, l_{y o}, l_{z o}\right)$, for it must iterate convergence node $J$ as illustrated in Fig. 3.

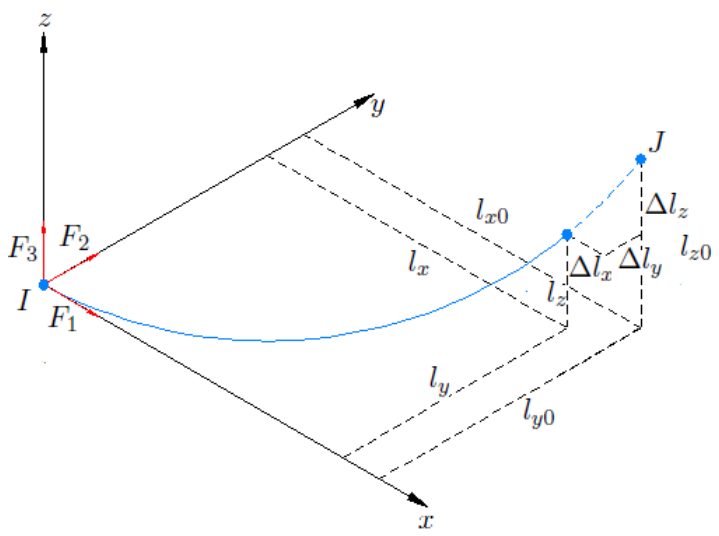

Fig. 3. Configuration in a Step of the iteration

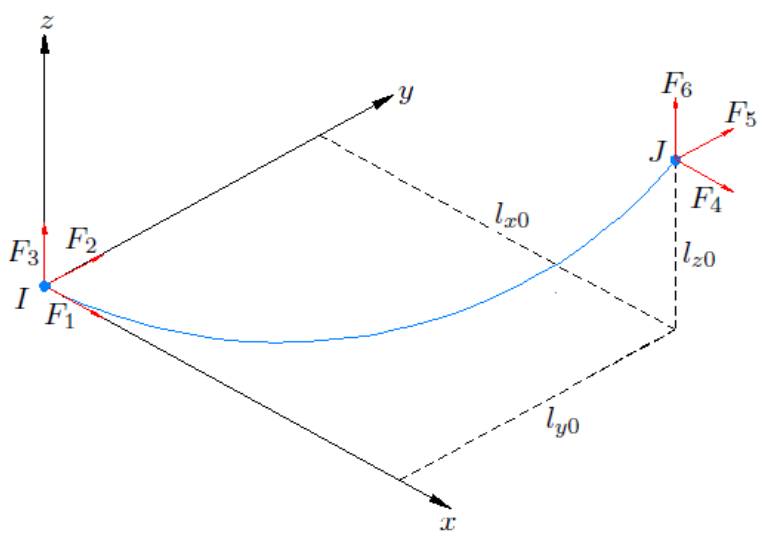

Fig. 4. Cable element after convergence. 


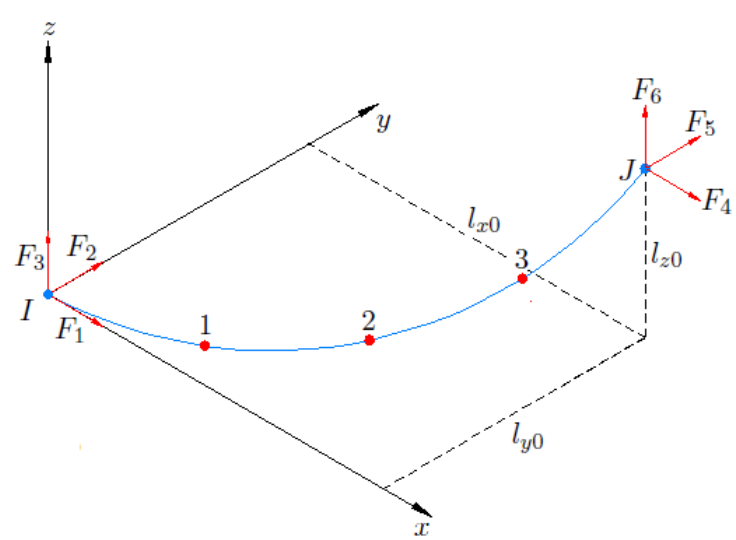

Fig. 5. Cable with internal nodes.

After satisfied convergence node $J$, can be calculated forces $\left(F_{4}, F_{5}, F_{6}\right)$ on that node using the equilibrium expressions (20).

Fig. 3 and Fig. 4 only show a cable element $I$ and $J$ ends which cannot be used when an external load is applied to a portion of the cable. Therefore, the cable is divided internally as a number of segments or against a known length, using the expressions (13), (14) and (15), as shown in Fig. 5.

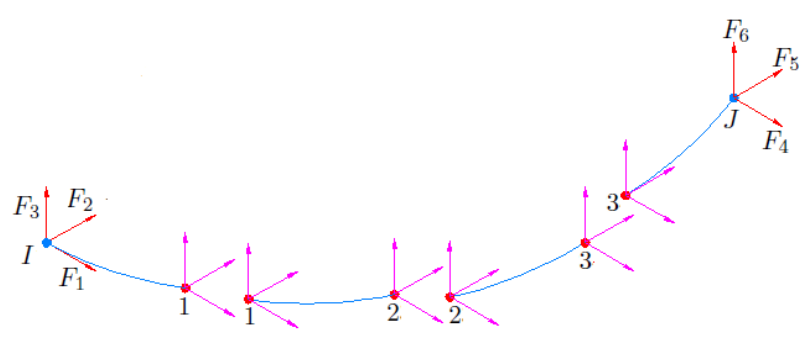

Fig. 6. Forces of each element.

Each segment is possible to determine the forces on the nodes added by equilibrium conditions as shown in Fig. 6 . These nodal forces and the length of each segment are used to determine the stiffness matrix of each cable segment.

The matrix of each cable segment is grouped into an overall matrix of the cable, which is used in the nonlinear analysis.

\section{E. Algorithm for Nonlinear Static Analysis}

The algorithms for solving mixed structure in a nonlinear condition are illustrated in Fig. 7 and Fig. 8.

\section{F. Nonlinear Dynamic Analysis}

It is resolved with Newmark method incrementally and iteratively. The iterative part is solved by the method of the secant stiffness (Secant method) or tangent stiffness (Newton method), and then the displacement, velocity and acceleration are updated in time $t+\Delta t$ as [13].

The mass matrix is concentrated and damping matrix is defined as:

$$
C=\alpha_{M} M+\alpha_{K} K
$$

where $\alpha_{M}$ and $\alpha_{K}$ are factors proportional to the mass damping and stiffness of the structure respectively, depending on the type of buffer that is supposed predominant (proportional to the mass, stiffness or both) [14].

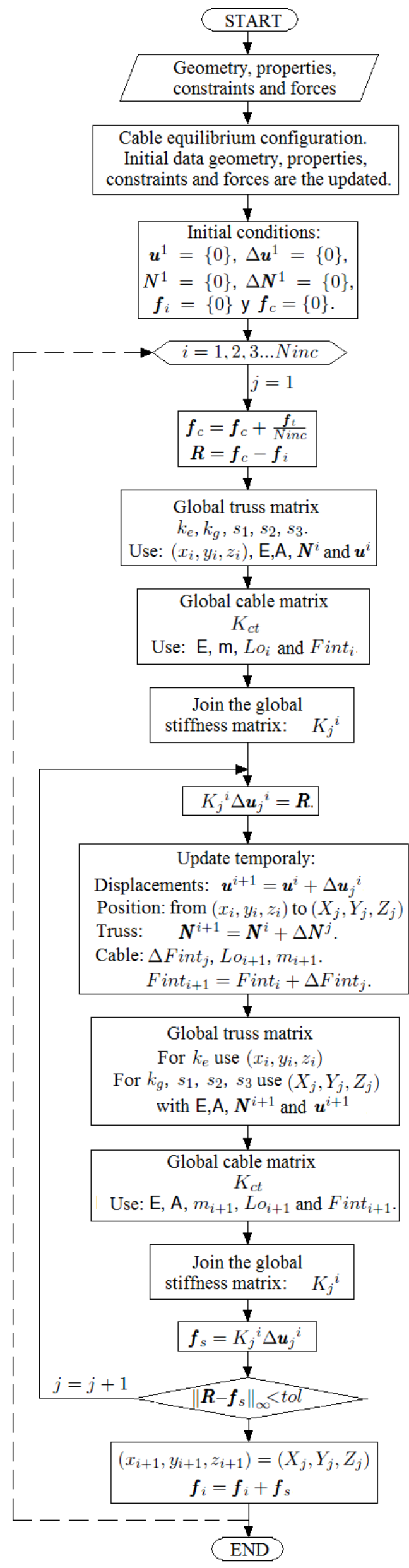

Fig. 7. Flow chart of Secant method. 


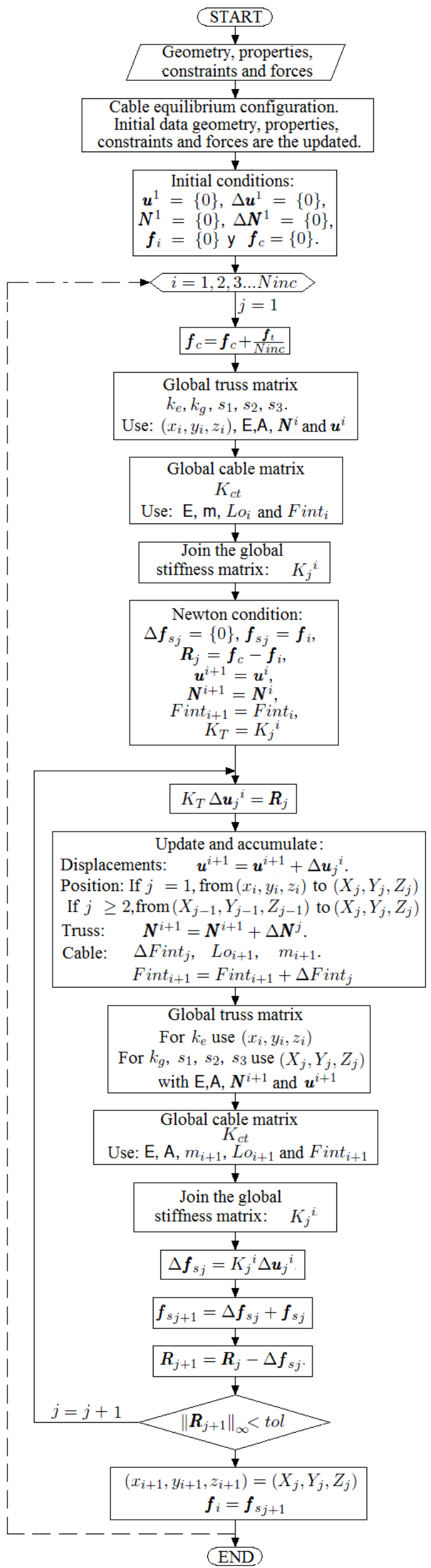

Fig. 8. Flow chart of Newton method.

\section{APPLICATIONS}

\section{A. Cable Suspended with Point Load}

The following application is taken as reference to validate different methods to simulate cables. Initially proposed by Michalos and Birnstiel, then analyzed by O'Brien and Francis (as indicated Huu [8]), Jayaraman and Knudson [11], Tibert [5], Andreu [6], Huu and Seung [8].

The problem is to determine the displacement of node 2 , when the prestressed cable under self-weight it is applied a concentrated load. The initial configuration and information are in Fig. 9 and Table I respectively.

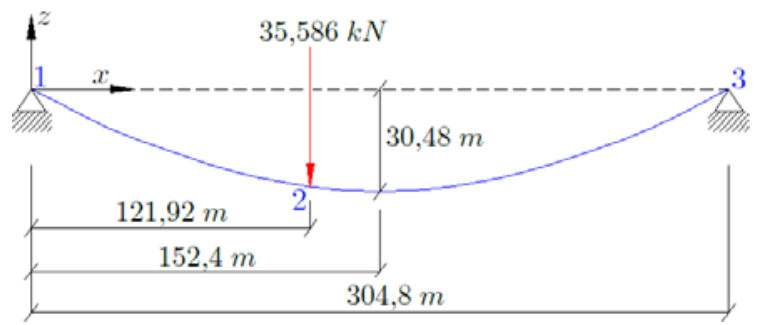

Fig. 9. Cable under self-weight and concentrated load.

TABLE I: INITIAL PROPERTIES OF CABLE SUSPENDED.

\begin{tabular}{lll}
\hline \hline & Item & Data \\
\hline $\mathrm{w}$ & Cable self-weight & $46.12 \mathrm{~N} / \mathrm{m}$ \\
$\mathrm{A}$ & Cross-sectional area & $5.484 \mathrm{~cm}^{2}$ \\
$\mathrm{E}$ & Elastic modulus & $13100.0 \mathrm{kN} / \mathrm{cm}^{2}$ \\
$\mathrm{Lo}_{1-2}$ & Unstressed cable length 1-2 & $125.85 \mathrm{~m}$ \\
$\mathrm{Lo}_{2-3}$ & Unstressed cable length 2-3 & $186.86 \mathrm{~m}$ \\
& Sag under self-weight at load point & $29.276 \mathrm{~m}$ \\
\hline \hline
\end{tabular}

The model used is composed of two cable elements, which have been derived from the initial configuration under self-weight. The concentrated load is applied incrementally to complete this load, with the ten-thousandth of the total load $(35,586 \mathrm{~N} / 10000)$ acceptable load increased.

Fig. 10, the Secant method is fast approaching the solution increases fewer and shorter convergence compared to put Newton.

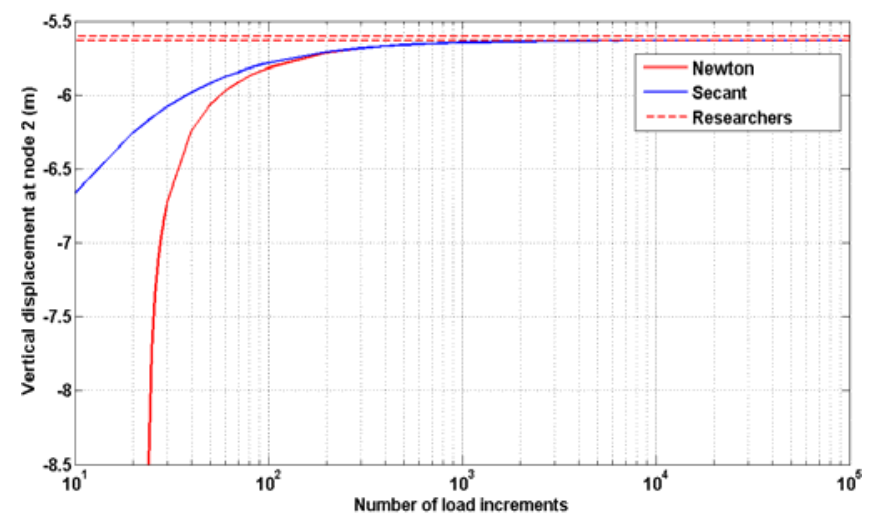

Fig. 10. Displacement - load increment curves.

Calculated displacements are acceptable when compared to the results of various researchers, as shown in Table II.

\section{B. Tridimensional Truss}

To verify the algorithm developed in space truss, analyze a section of tower height $3.00 \mathrm{~m}$ subject to a lateral load of $1000 \mathrm{~kg}$. 
TABLE II: COMPARISON OF DISPLACEMENT NODE 2.

\begin{tabular}{llcc}
\hline \hline \multirow{2}{*}{ Researcher } & \multirow{2}{*}{ Element type } & \multicolumn{2}{c}{ Displacements (m) } \\
\cline { 3 - 4 } & & Vertical & Horizontal \\
\hline Michalos and Birnstiel [8] & Elastic straight & -5.472 & -0.845 \\
O'Brien [8] & Elastic catenary & -5.627 & -0.860 \\
Jayaraman and Knudson [11] & Elastic catenary & -5.626 & -0.859 \\
Tibert [5] & Elastic parabola & -5.601 & -0.866 \\
Tibert [5] & Elastic catenary & -5.626 & -0.859 \\
Andreu [6] & Elastic catenary & -5.626 & -0.860 \\
Huu and Seung [8] & Elastic catenary & -5.626 & -0.859 \\
Present work & Elastic catenary & -5.627 & -0.860 \\
\hline \hline
\end{tabular}

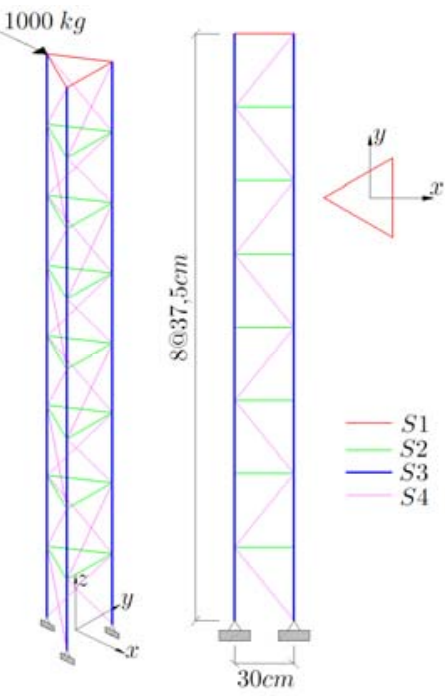

Fig. 11. Tridimensional truss.

This model is also used in [15] to verify the difference between the two programs. The geometric configuration and physical properties are presented in Fig. 11 and Table III respectively.

TABLE III: PROPERTIES.

\begin{tabular}{ccccc}
\hline \hline Section & Type & $\begin{array}{c}\text { Area } \\
\left(\mathrm{cm}^{2}\right)\end{array}$ & $\begin{array}{c}E \\
\left(\mathrm{~kg} / \mathrm{cm}^{2}\right)\end{array}$ & $\begin{array}{c}\text { Density } \\
\left(\mathrm{kg} / \mathrm{cm}^{3}\right)\end{array}$ \\
\hline$S_{1}$ & L2"x2"x3/16" & 4.62 & 2038902 & 0.00785 \\
$S_{2}$ & L1.5"x1.5"x3/16" & 3.40 & 2038902 & 0.00785 \\
$S_{3}$ & PX1.5" & 6.90 & 2038902 & 0.00785 \\
$S_{4}$ & $\varnothing 5 / 8 "$ & 1.98 & 2038902 & 0.00785 \\
\hline \hline
\end{tabular}

The first analysis is linear, for this global elastic stiffness matrix is resolved in a single load increase, while the nonlinear analysis is performed in increments of ten-thousandth of the total external load $(1000 \mathrm{~kg} / 10000)$.

If we use as parameter the required number of increments of charge, the secant method approximates faster to the solution, as shown in Fig. 12.
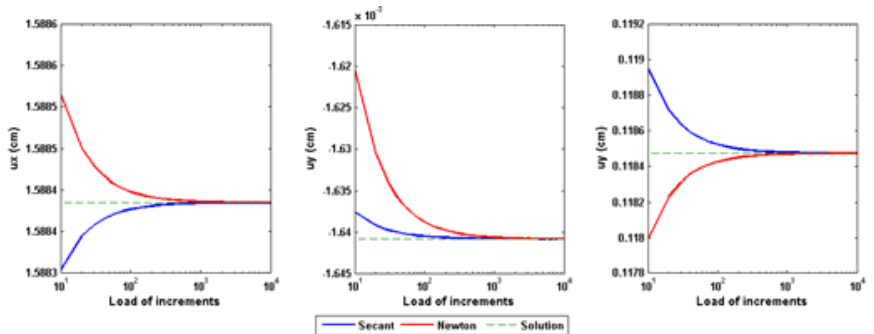

Fig. 12. Displacement - load increment curves in nonlinear analysis at node loaded.

\section{Tridimensional Truss with Stayed Cables}

The following application aims to engage a space truss with stay cables into a single structure, which then determine their displacement under self-weight and when the cable is prestressed at the bottom with $30 \mathrm{~kg}$, we apply a lateral load of $1000 \mathrm{~kg}$. This structure is also analyzed with [15] program to verify the nodal displacements.

Another aim is to verify the amount necessary to obtain more accurate results in solving cable elements.

The physical properties of the reinforcement element are similar to those of the application B, while the properties of the cable element are found in Table IV. The model used is shown in Fig. 13.

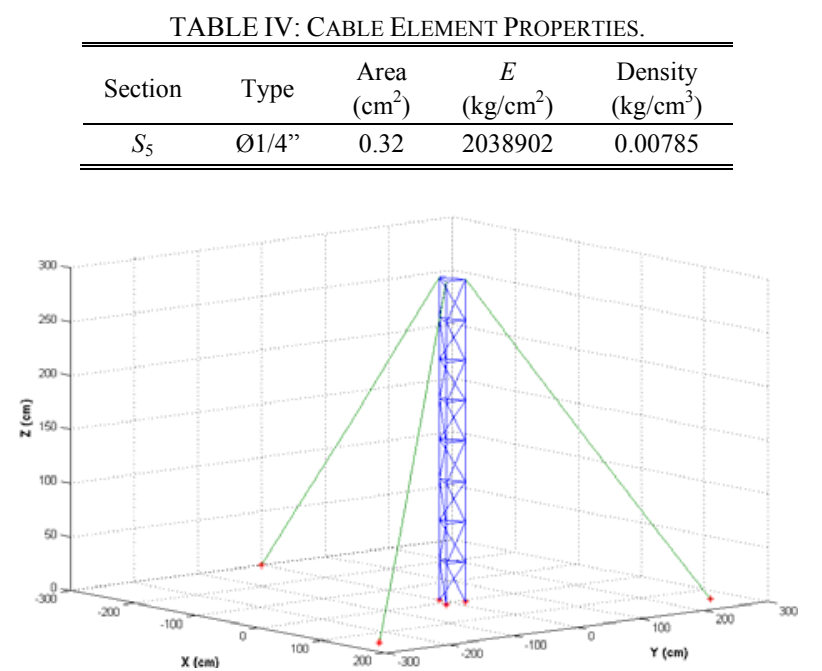

Fig. 13. Tridimensional truss with stayed cables.

Nonlinear analysis by self-weight and the prestressed cable, is made in increments of one-hundredth of the load (self-weight/100), whereby the deformation shown in Fig. 14. It is noted that these displacements are similar when the cable edge is subdivided into several elements before applying the load side.

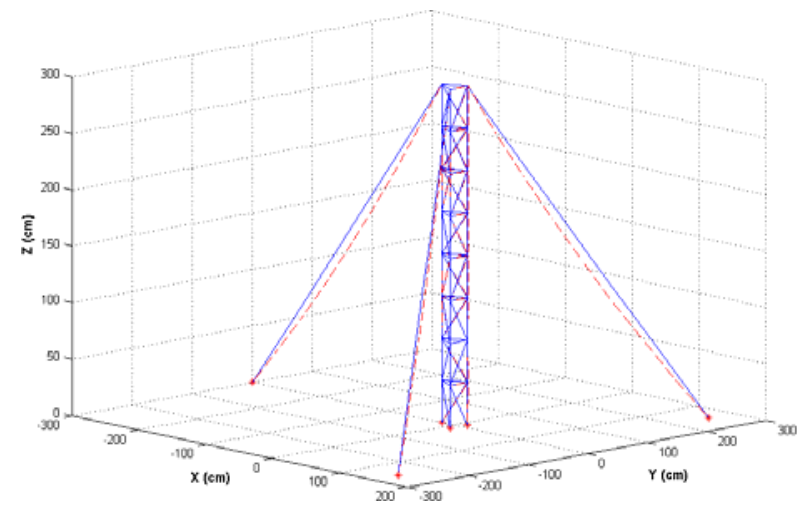

Fig. 14. Geometry deformed by self-weight of tridimensional truss with stayed cables (not to scale).

When put side loading $1000 \mathrm{~kg}$, loaded displacement for various branches of the cable element node is recorded, as shown in Fig. 15, in order to determine the required amount of cable elements. With this figure, it is showed this trend to employ more than 10 cable divisions. It is noted that as more cable elements add to it, this differs more with [15] program results. 

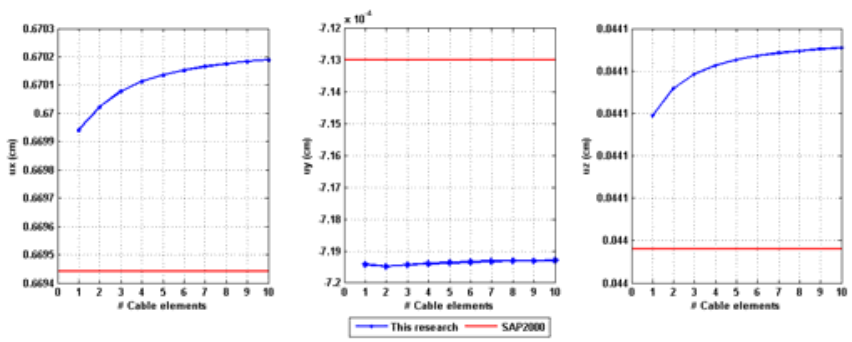

Fig. 15. Displacement at three directions with different amounts of cable elements.

\section{Plane Cable Net}

The structure considered is the cable net as shown in Fig. 16, whose physical properties are listed in Table V.

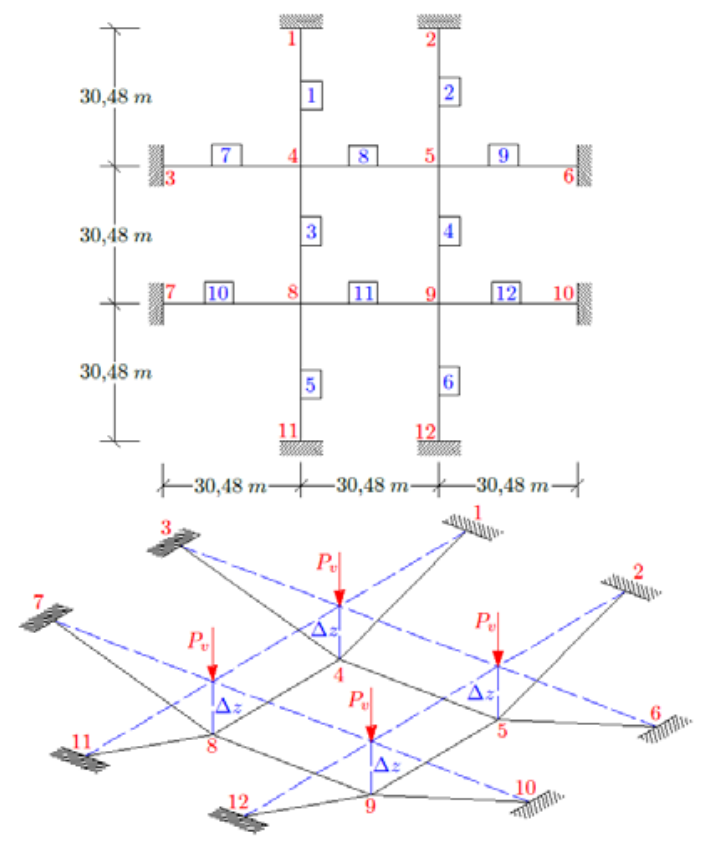

Fig. 16. Prestressed cable net under vertical loads.

TABLE V: CABLE INFORMATION

\begin{tabular}{lll}
\hline \hline & Item & Data \\
\hline$w$ & Cable self-weight & $1.459 \mathrm{~N} / \mathrm{m}$ \\
$A$ & Cross-sectional area & $146.45 \mathrm{~mm}^{2}$ \\
$E$ & Elastic modulus & $82.74 \mathrm{Mpa}$ \\
$T o_{H}$ & Prestressing force of horizontal members & $24.28 \mathrm{kN}$ \\
$T o_{I}$ & Prestressing force of horizontal members & $23.69 \mathrm{kN}$ \\
$P v$ & Load point & $35.59 \mathrm{kN}$ \\
$\Delta z$ & Assumed initial configuration & $9.144 \mathrm{~m}$ \\
\hline \hline
\end{tabular}

In the stretched configuration of the Fig. 16, the vertical load $P v$ are applied to the nodes 4, 5, 8 and 9. The analysis is performed for load increments of thousandths of $P v$ $(P v / 1000)$. The results of the displacement at node 8 are listed in Table VI.

\section{TABLE V: DisPlacement AT NODE 8}

\begin{tabular}{lcccc}
\hline \hline \multicolumn{1}{c}{ Researcher } & Element type & $u_{x}(\mathrm{~mm})$ & $u_{x}(\mathrm{~mm})$ & $u_{x}(\mathrm{~mm})$ \\
\hline Jayaraman and Knudson & Elastic catenary & -39.62 & -40.20 & -446.32 \\
[12] & Elastic parabola & -40.78 & -40.78 & -453.33 \\
Tibert [5] & Elastic catenary & -40.78 & -40.78 & -450.04 \\
Tibert [5] & Elastic catenary & -40.13 & -40.13 & -446.50 \\
Huu and Seung [8] & Elastic catenary & -40.47 & -40.47 & -449.46 \\
SAP2000 [15] & Elastic catenary & -40.53 & -40.53 & -450.12 \\
Present work & & & & \\
\hline \hline
\end{tabular}

\section{E. Tridimensional Dynamic Truss}

Armor with 72 truss elements is shown in Fig. 17 in order to verify the effectiveness of predicting the nonlinear effects when subjected to seismic acceleration record El Centro (1940). This application is shown by Huu [16] and the values of its physical properties are presented in Table VII.

\begin{tabular}{lll}
\multicolumn{3}{c}{ TABLE VII: TRIDIMENSIONAL TRUSS INFORMATION } \\
\hline \hline & Item & Data \\
\hline$A$ & Cross-sectional area & $38.485 \mathrm{~cm}^{2}$ \\
$E$ & Elastic modulus & $200 \mathrm{GPa}$ \\
$\mathrm{M}$ & Lumped masses & $100 \mathrm{kN} \mathrm{s} / \mathrm{m}$ \\
$\xi_{i}, \xi_{j}$ & Fraction of critical damping & 0.05 \\
\hline \hline
\end{tabular}

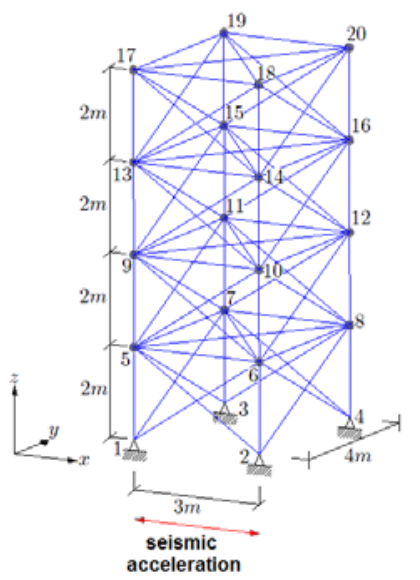

Fig. 17. Space truss with concentrated masses.

Fig. 18 shows the displacement responses at node 19 in $x$ direction. From this figure and Table VIII, differences in peak displacements are less than $0.46 \%$, which reflects a low discrepancy between Huu and the present work.

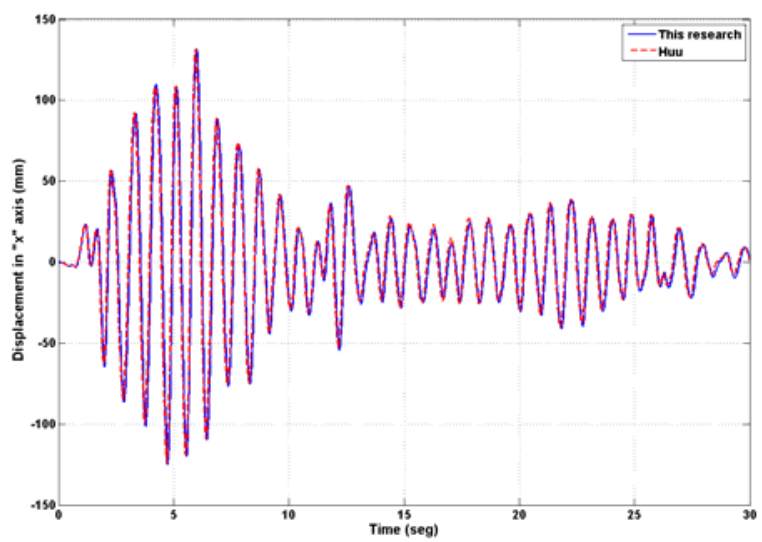

Fig. 18. Displacement response at node 19.

\begin{tabular}{lccc}
\multicolumn{4}{c}{ TABLE VIII: COMPARISON OF PEAK DISPLACEMENT } \\
\hline \hline Huu [16] & Present work & Difference (\%) \\
\hline Max & 132.21 & 131.60 & 0.46 \\
Min & -124.46 & -124.85 & 0.31 \\
\hline \hline
\end{tabular}

\section{CONCLUSIONS}

It has demonstrated the possibility of coupling cable and truss elements in a structure, and consider the nonlinearity of these elements, it has been used for nonlinear Variational Formulation given by Yang [9], which allows to deduce 
matrices including elastic, geometric and higher order effects of a truss element, while for the cable element, duly verified from analytical equations, allows to deduce nonlinear matrices.

Also in structures composed of cable elements, the secant method is best approximates the solution when having less load increases (see Fig. 10) while in structures formed by truss elements, the secant method is closer to the solution when load increases are becoming smaller.

The results obtained from the elements and the proposed methodology, show agreement with the results obtained with commercial programs and those results reported by other researchers, as noted in Section III.

\section{REFERENCES}

[1] A. Pintea, "Comparison between the linear and nonlinear responses of cable structures I - static loading," Civil Engineering and Architecture, vol. 55 , no. 2, pp. 182-188, 2012.

[2] M. Cuomo and L. Greco, A Finite Element Cable for the Analysis of Cable Nets, Faculty of Engineering, University of Catania, Ancona, 2009.

[3] A. Kwan "A new approach to geometric nonlinearity of cable structures," Computer and Structures, vol. 67, pp. 243-352, 1998.

[4] M. Irvine, Cable Structure, MIT Press, Cambridge, 1982.

[5] G. Tibert, Numerical analyses of cable roofs structures, Licentiate Thesis, Department of Structural Engineering, Royal Institute of Technology, Estocolmo, 1998.

[6] A. Andreu, L. Gil and P. Roca, "A new deformable catenary element for the analysis of cable net structures," Computer and Structures, vol. 84 , pp. $1882-1890,2006$.

[7] W. Ren, M. Huang, and W. Hu, "A parabolic cable element for static analysis of cable structures," International Journal for Computer Aided Engineering and Software, vol. 25, no. 4, pp. 366-384, 2008.

[8] T. Huu and K. Seung, "Nonlinear static and dynamic of cable structures," F inite Elements in Analysis and Design, vol. 47, pp. 237-246, 2011.
[9] B. Yang, Theory and Analysis of Nonlinear Framed Structures, Prentice Hall, Singapore, 1994.

[10] M. Pallares and C. Rodriguez, "Validación de la formulación numérica de la catenaria elástica con Ansys," Escuela Regional de Matemáticas, Universidad del Valle, Cali, XVI, 1, pp. 63-85, 2008.

[11] H. Jayaraman and W. Knudson, "A curved element for the analysis of cable structure," Computer and Structures, vol. 14, pp. 325-333, 1981.

[12] R. Karoumi, "Some modeling aspects in the nonlinear finite element analysis of cable supported bridges," Computer and Structures, vol. 71, pp. 397-412, 1999.

[13] A. Chopra, Dynamics of Structures, Second edition, Prentice Hall, N. Y., 1995.

[14] IDARC 2D Version 4.0: A Program for the Inelastic Damage Analysis of Buildings - Technical Report NCEER-96-0010, University at Buffalo, N. Y., 1996.

[15] SAP2000, CSI Analysis Reference Manual, Computer and Structures, Berkeley, 2010.

[16] T. Huu and K. Seung, "Practical advanced analysis software for nonlinear dynamic analysis of steel structures," Journal of Constructional Steel Research, vol. 67, pp. 453-461, 2011.

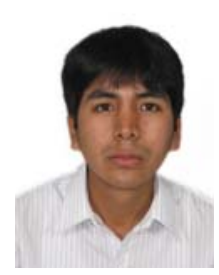

Ever Coarita was born in Puno, 1988. He was a professional engineer from the National University of Engineering, Lima, Peru. At present, he is a professor of mathematics courses in the Faculty of Civil Engineering at National University of Engineering.

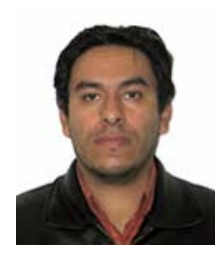

Leonardo Flores was born in Cuzco, 1968. He was a professional engineer from the National University of Engineering, Lima, Peru. He received the grade of master of science in structures and transport engineering at University National of Engineering, Lima, Peru. At present, He is a professor of mathematics courses in the Faculty of Civil Engineering at National University of Engineering. 